\section{Case Reports in Oncology}

\title{
Inflammatory Myofibroblastic Tumor of the Urinary Bladder: A Case Report
}

\author{
Toshiki Etani $^{a, b} \quad$ Taku Naiki $^{b} \quad$ Takashi Nagai $^{b} \quad K^{b i t a r o ~ I i d a ~}{ }^{b}$ \\ Ryosuke Ando $^{b}$ Aya Naiki-Ito ${ }^{c}$ Noriyasu Kawai ${ }^{b} K$ Keiichi Tozawa ${ }^{b}$ \\ Kentaro Mizuno $^{b} \quad$ Atsushi Okada $^{b}$ Tohru Mogami $^{a} \quad$ Takahiro Yasui $^{b}$ \\ ${ }^{a}$ Department of Urology, JA Mie Komono Kosei Hospital, Komono, Japan; ${ }^{b}$ Department of \\ Nephro-urology, Nagoya City University, Graduate School of Medical Sciences, Nagoya \\ City, Japan; 'Department of Experimental Pathology and Tumor Biology, Nagoya City \\ University, Graduate School of Medical Sciences, Nagoya City, Japan
}

\section{Keywords}

Inflammatory myofibroblastic tumor - Bladder · Anaplastic lymphoma kinase - Vascular endothelial growth factor

\begin{abstract}
An inflammatory myofibroblastic tumor (IMT) is a distinctive neoplasm composed of myofibroblastic and fibroblastic spindle cells, accompanied by inflammatory infiltration of plasma cells, lymphocytes, and eosinophils. IMTs rarely occur in the urinary bladder. It is important to distinguish this tumor from other malignant spindle cell tumors. Herein, we report a patient with an IMT showing muscle invasion, who underwent a transurethral resection of the bladder tumor and, at a later date, partial cystectomy. The resected tumor specimen revealed a proliferation of spindle-shaped cells on a background of plasma cells and lymphocytes. Immunohistochemical staining showed the tumor to be positive for anaplastic lymphoma kinase (ALK), smooth muscle actin, and vascular endothelial growth factor (VEGF). Such histopathological findings were indicative of an IMT, suggesting the use of inhibitors of ALK and VEGF as pharmacotherapy.




\section{Introduction}

An inflammatory myofibroblastic tumor (IMT) is a distinctive neoplasm composed of myofibroblastic and fibroblastic spindle cells accompanied by inflammatory infiltration of plasma cells, lymphocytes, and eosinophils [1]. IMTs rarely occur in the urinary bladder. It is important to distinguish this tumor from other malignant spindle cell tumors, such as the sarcomatoid variant of urothelial carcinoma and leiomyosarcoma. We report a case of IMT of the urinary bladder in a 52-year-old male diagnosed by transurethral resection of the bladder tumor (TURBT) treated by partial cystectomy.

\section{Case Presentation}

The patient was a 52-year-old male who presented with asymptomatic gross hematuria. His medical history revealed a diagnosis of rheumatoid arthritis 3 years ago, and prednisolone $10 \mathrm{mg} /$ day was subsequently prescribed. Cystoscopy revealed a solitary nonpapillary tumor with surrounding edema at the dome of the bladder (fig. 1a). Enhanced computed tomography (CT) and magnetic resonance imaging (MRI) revealed an early enhancing bladder tumor infiltrating the outside of the bladder muscle layer (fig. 1). There was no metastasis.

He subsequently underwent TURBT. Intraoperative findings showed a large solid bladder mass, measuring approximately $3 \mathrm{~cm}$ from the dome to the anterior wall of the bladder. Its smooth surface was not typical of urothelial carcinoma. We resected the tumor to the depth of the muscle layer, but normal muscle tissue was not seen. We consequently diagnosed this tumor as a muscle-invasive bladder tumor.

Histopathology results were consistent with an IMT. The bladder tissue, including muscle, was widely infiltrated by spindle cells in a myxoid stroma accompanied by infiltration of inflammatory cells (fig. 2a, b). Moderate nuclear atypia was confirmed in the cells. Mitotic activity was inconspicuous, with no atypical mitotic figures identified. Immunohistochemical staining was positive for vimentin and smooth muscle actin (fig. 2c) and negative for AE1/AE3, desmin, myogenin, S-100, CD34, c-kit, CD68, and bcl-2. MIB-1 positivity was maximally estimated to be approximately $2 \%$ (data not shown). Conclusively, we diagnosed this tumor as an IMT with muscle layer infiltration.

We subsequently performed a partial cystectomy. The excised specimen is shown in figure $2 \mathrm{~d}$. A pathological examination of the excised specimen revealed the proliferation of spindle cells expanding into the bladder musculature, accompanied by inflammatory cell infiltration. There was no neoplastic urothelium. A conclusive diagnosis of this tumor as an IMT was made. For the assessment of therapeutic options, we measured the expressions of anaplastic lymphoma kinase (ALK), vascular endothelial growth factor (VEGF), and cyclooxygenase 2 (COX2), known as growth factors involved in tumor proliferation. Immunohistochemical staining was positive for ALK and VEGF and negative for COX2 (fig. 3). To date, the patient has been followed for 3 years without any evidence of recurrence.

\section{Discussion}

The first report of an IMT of the urinary bladder was made in 1980 [2]. It is characterized by atypical spindle cell proliferation and inflammatory cell infiltrates primarily involv- 
ing lymphocytes and plasma cells. Although it has been associated with trauma, surgery, and infection, the majority of IMT cases occur spontaneously. IMT tumors are classified as intermediate (rarely metastasizing) tumors according to the WHO classification of soft tissue tumors. IMTs occur in the mesentery, omentum, retroperitoneum, pelvis, and abdominal soft tissues in 73\% of cases [1]. However, the occurrence of an IMT in the urinary bladder is unusual. A systematic review by Teoh et al. [3] evaluated 182 IMT cases and found a mean age of patients of 38.9 years, with a predilection for females.

IMTs resemble malignant spindle cell tumors, such as sarcomatoid carcinoma, leiomyosarcoma, or rhabdomyosarcoma, making diagnosis difficult [4]. Recent reports have indicated that ALK, which was originally identified as a protein overexpressed in anaplastic largecell lymphomas, was also overexpressed in a substantial proportion of IMTs [5, 6]. A positive finding of ALK by immunohistochemistry in up to $87.5 \%$ of IMTs can be useful for the differentiation of IMTs from other spindle cell tumors. In this case, ALK immunohistochemistry was positive and useful for a definite final diagnosis.

$A L K$ gene translocation in IMTs has also been reported, for which detection by fluorescence in situ hybridization (FISH) is useful. However, performing FISH in paraffin-embedded specimens is questionable for obtaining a precise diagnosis. ALK protein expression, as determined by immunohistochemistry, correlates well with the presence of a rearrangement in the $A L K$ gene $[7,8]$. Therefore, we did not undertaken FISH analysis in this case because of the strong immunohistochemical positivity of ALK.

In the consideration of a therapy for IMT, surgical resection is first recommended. In a systematic review undertaken in 2014, most patients underwent TURBT (60.8\%); others had partial $(29.2 \%)$ and radical cystectomy $(9.2 \%)$, and 5 patients experienced local recurrence [3]. Complete surgical resection is important to avoid local recurrence [9]. While partial or radical cystectomy ensures complete resection of IMT, TURBT is also a considerable choice, given the benign course of an IMT. In the present case, CT, MRI, and the examination of a TURBT indicated a muscle layer-infiltrating lesion, so we performed a partial cystectomy. There were no reports as to the safety margin of IMT in the bladder at partial cystectomy. We performed the operation by setting a margin of about $1 \mathrm{~cm}$, and there was no sign of local recurrence in the bladder wall. For performing the operation while keeping the bladder capacity, further investigation as to the surgical margin should be made in the future.

For a nonresectable IMT, pharmacotherapy has also been reported. COX2 and VEGF expression have been detected in IMTs and are thought to be therapeutic targets [10]. Additionally, as an anti-inflammatory drug, COX2 inhibitors used for nonresectable IMTs have often been reported [11]. An ALK inhibitor, crizotinib, has also been used in the treatment of IMTs [12]. In the present case, we examined COX2, VEGF, and ALK protein expression with immunohistochemistry and found IMT tissues to be VEGF and ALK positive. Prednisolone has previously been prescribed in this case. We also thought that the tumor was resectable by partial cystectomy and therefore decided against a course of pharmacotherapy. However, in the case of recurrence when the tumor is not resectable, pharmacotherapy may become a viable therapeutic option.

In conclusion, an IMT is a rare neoplasm of unknown malignant potential. A typical IMT can be locally aggressive and may require radical surgical resection; close follow-up is therefore warranted. 


\section{Statement of Ethics}

Written informed consent was obtained from the patient for publication of this case report and accompanying images. A copy of the written informed consent is available for review from the Editor-in-Chief of this journal. This study was conducted according to the Declaration of Helsinki.

\section{Disclosure Statement}

The authors declare that they have no potential conflicts of interest.

\section{References}

1 Fletcher CDM, Bridge JA, Hogendoorn P, Mertens F: WHO Classification of Tumours of Soft Tissue and Bone. Lyon, IARC Press, 2013, vol 5, pp 83-84.

2 Roth J: Reactive pseudosarcomatous response in urinary bladder. Urology 1980;16:635-637.

-3 Teoh JY, Chan NH, Cheung HY, Hou SS, Ng CF: Inflammatory myofibroblastic tumors in urinary bladder: a systematic review. Urology 2014;84:503-508.

-4 Machioka K Kitagawa Y, Izumi K, Kitamura S, Ikeda H, Namiki M: Inflammatory myofibroblastic tumor of the urinary bladder with benign pelvic lymph node enlargement: a case report. Case Rep Oncol 2014;7:571-575.

-5 Tsuzuki T, Magi-Galluzzi C, Epstein JI: ALK-1 expression in inflammatory myofibroblastic tumor of the urinary bladder. Am J Surg Pathol 2004;28:1609-1614.

6 Rao RN, Ranjan P, Singla N, Pandey R: Inflammatory myofibroblastic tumor of the urinary bladder diagnosed by anaplastic lymphoma kinase immunostaining. Urol Ann 2012;4:115-118.

7 Freeman A, Geddes N, Munson P, Joseph J, Ramani P, Sandison A, Fisher C, Parkinson MC: Anaplastic lymphoma kinase (ALK1) staining and molecular analysis in inflammatory myofibroblastic tumours of the bladder: a preliminary clinicopathological study of nine cases and review of the literature. Mod Pathol 2004;17:765-771.

-8 Sukov WR, Cheville JC, Carlson AW, Shearer BM, Piatigorsky EJ, Grogg KL, Sebo TJ, Sinnwell JP, Ketterling RP: Utility of ALK-1 protein expression and ALK rearrangements in distinguishing inflammatory myofibroblastic tumor from malignant spindle cell lesions of the urinary bladder. Mod Pathol 2007;20:592-603.

-9 Wei L, Jianbo L, Qiang W, Hai Y, Zhixiang L: Inflammatory myofibroblastic tumour of the bladder: case report and review of the literature. Can Urol Assoc J 2013;7:E237-E240.

10 Applebaum H, Kieran MW, Cripe TP, Coffin CM, Collins MH, Kaipainen A, Laforme A, Shamberger RC: The rationale for nonsteroidal anti-inflammatory drug therapy for inflammatory myofibroblastic tumors: a children's oncology group study. J Pediatr Surg 2005;40:999-1003.

11 Chavez C, Hoffman MA: Complete remission of ALK-negative plasma cell granuloma (inflammatory myofibroblastic tumor) of the lung induced by celecoxib: a case report and review of the literature. Oncol Letter 2013;5:1672-1676.

12 Butrynski JE, D’Adamo DR, Hornick JL, Dal Cin P, Antonescu CR, Jhanwar SC, Ladanyi M, Capelletti M, Rodig SJ, Ramaiya N, Kwak EL, Clark JW, Wilner KD, Christensen JG, Jänne PA, Maki RG, Demetri GD, Shapiro GI: Crizotinib in ALK-rearranged inflammatory myofibroblastic tumor. N Engl J Med 2010;18:1727-1733. 


\section{Case Reports in Oncology}
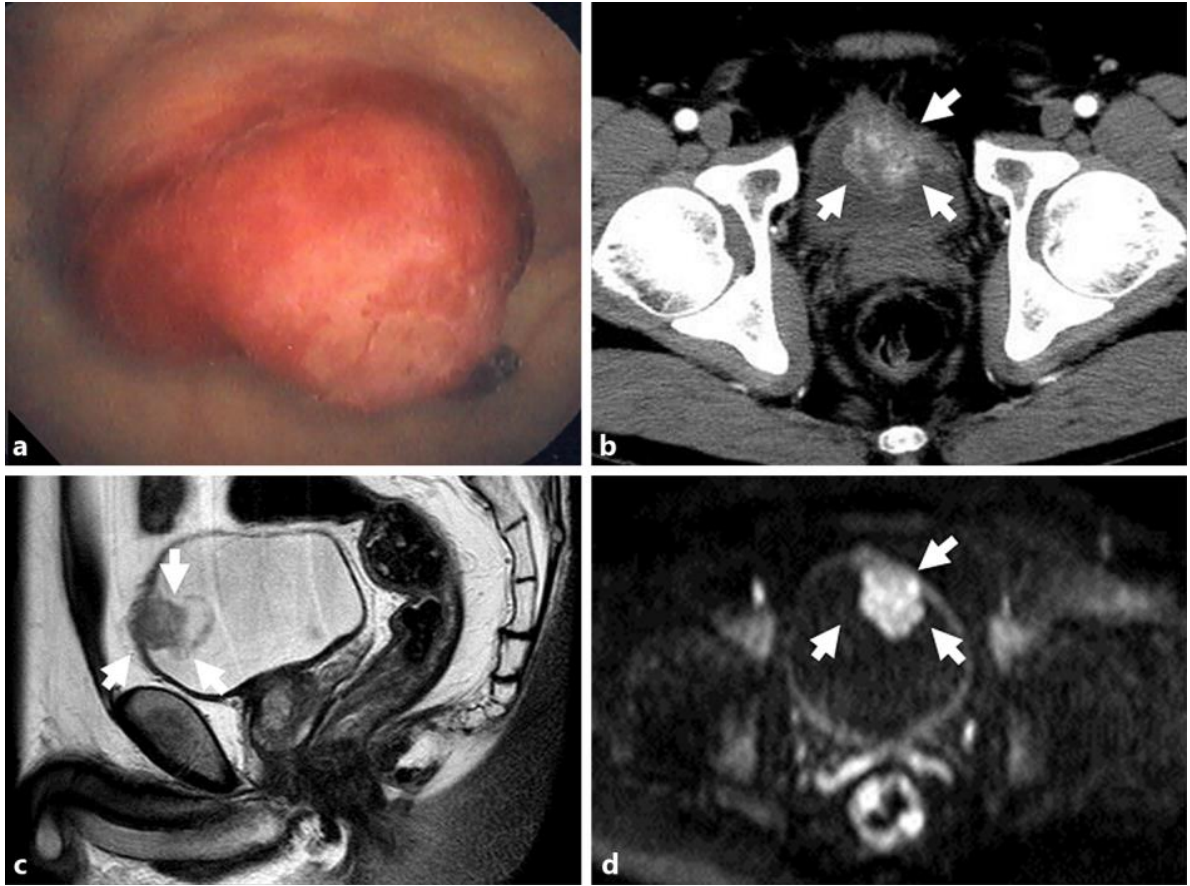

Fig. 1. a Cystoscopy revealed a solitary nonpapillary tumor with surrounding edema at the dome of the bladder. b Enhanced CT showed an early enhancing bladder tumor (white arrows). c, d MRI revealed an early enhancing bladder tumor (white arrows) infiltrating the outside of the bladder muscle layer. A T2weighted image (c) and a diffusion-weighted image (d) are shown. 


\section{Case Reports in Oncology}

\begin{tabular}{l|l}
\hline Case Rep Oncol 2016;9:464-469 \\
\hline $10.1159 / 000448550$ & $\begin{array}{l}\text { C 2016 The Author(s). Published by S. Karger AG, Basel } \\
\text { www.karger.com/cro }\end{array}$ \\
\hline
\end{tabular}
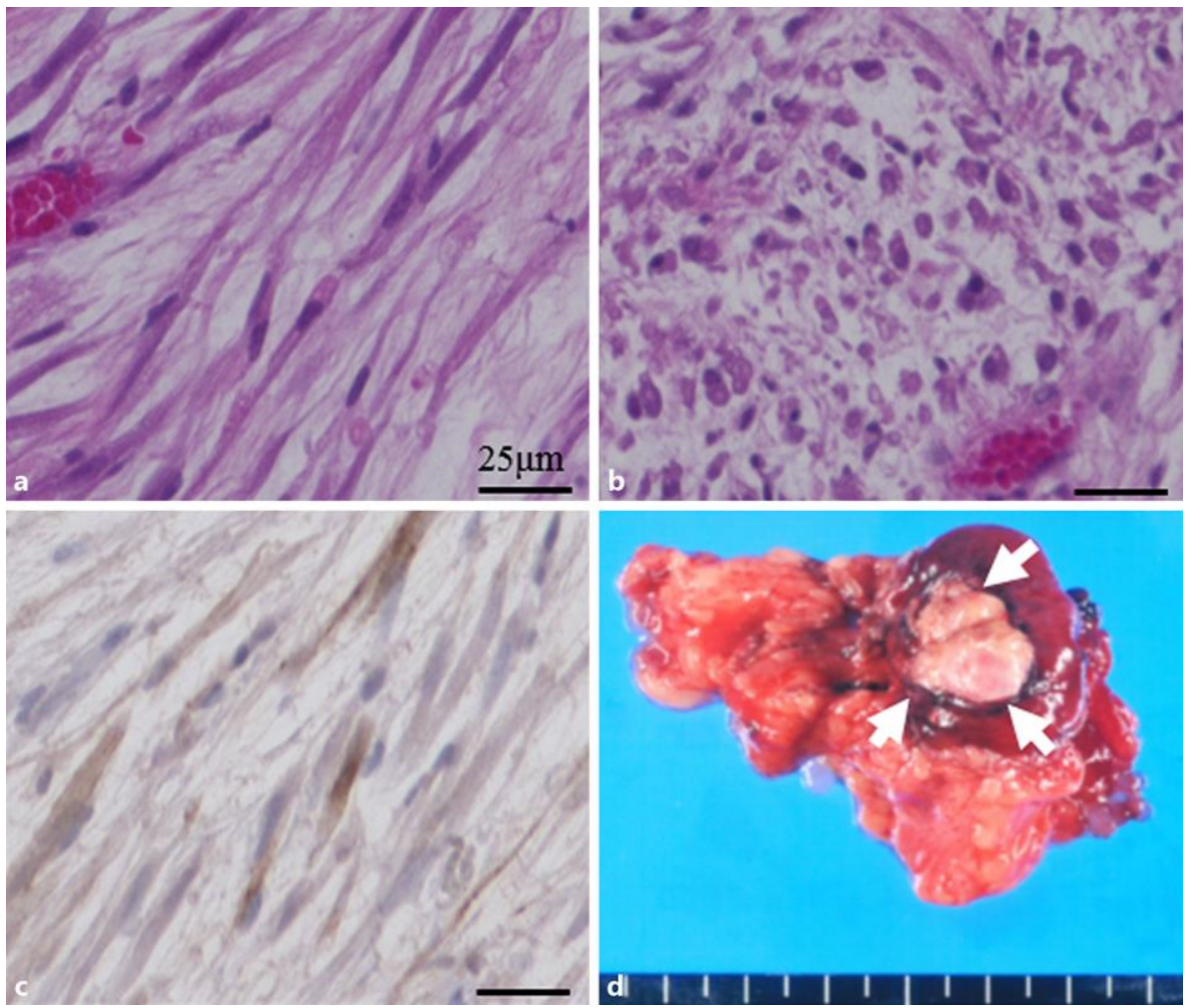

Fig. 2. a, b Bladder tissue, including muscle, was widely infiltrated by spindle cells (a) in a myxoid stroma accompanied by an inflammatory cell infiltrate (b) in hematoxylin and eosin stains. c An immunohistochemical test of the resected tumor showed positivity for smooth muscle cell actin. $\mathbf{d}$ The excised specimen of a partial cystectomy. The tumor is indicated by white arrows.
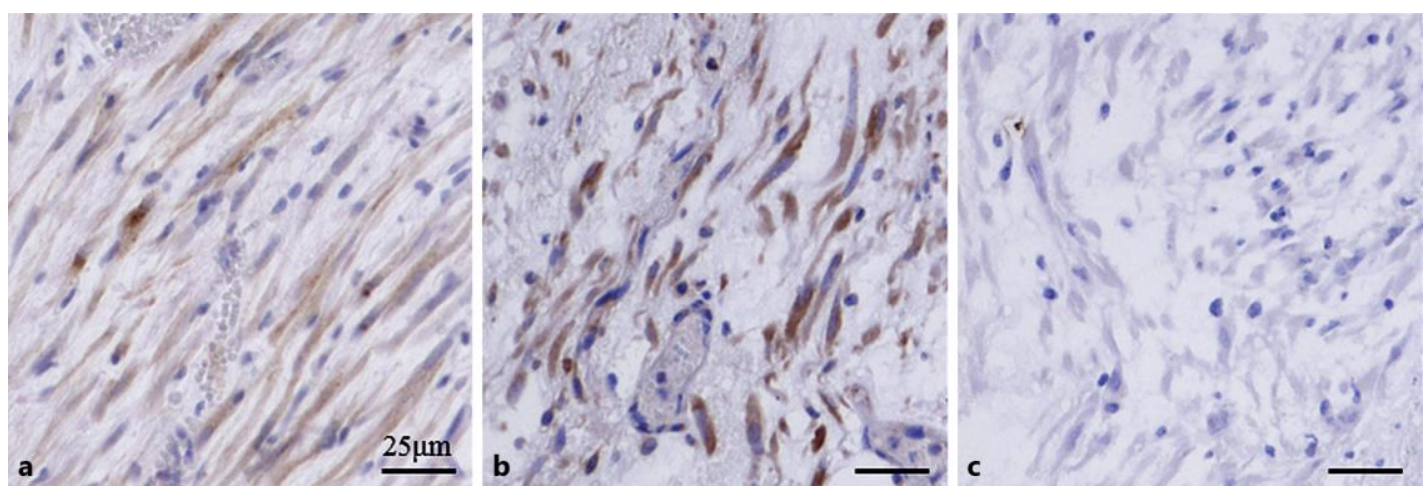

Fig. 3. Immunohistochemical tests for ALK (a), VEGF (b), and COX2 (c). 